\title{
openheart Strengthening effects of bone marrow mononuclear cells with intensive atorvastatin in acute myocardial infarction
}

\author{
Yue-Jin Yang (D , ${ }^{1}$ Hai-Yan Qian, ${ }^{1}$ Lei Song, ${ }^{1}$ Yong-Jian Geng, ${ }^{2}$ Run-lin Gao, ${ }^{1}$ \\ $\mathrm{Na} \mathrm{Li},{ }^{3}$ Hong Wang, ${ }^{4}$ Xia-Qiu Tian, ${ }^{4} \mathrm{Ji}$ Huang, ${ }^{5}$ Pei-Sen Huang, ${ }^{1}$ Jun $\mathrm{Xu},{ }^{1}$ \\ Rui Shen, ${ }^{6}$ Min-Jie Lu, ${ }^{7}$ Shi-Hua Zhao, ${ }^{7}$ Wei-Chun Wu, ${ }^{8}$ Yuan Wu, ${ }^{1}$ Jun Zhang, ${ }^{1}$ \\ Jie Qian, ${ }^{1}$ Jun-Yan Xu, ${ }^{1}$ Yu-Yan Xiong ${ }^{1}$
}

\begin{abstract}
- Additional material is published online only. To view please visit the journal online (http://dx.doi.org/10.1136/ openhrt-2019-001139)
\end{abstract}

To cite: Yang Y-J, Qian H-Y, Song $L$, et al. Strengthening effects of bone marrow mononuclear cells with intensive atorvastatin in acute myocardial infarction. Open Heart 2020;7:e001139. doi:10.1136/ openhrt-2019-001139

Y-JY and $\mathrm{H}-\mathrm{YQ}$ contributed equally.

Received 19 July 2019 Revised 20 February 2020 Accepted 26 March 2020

Check for updates

(C) Author(s) (or their employer(s)) 2020. Re-use permitted under CC BY. Published by BMJ.

For numbered affiliations see end of article.

Correspondence to Yue-Jin Yang; yangyjfw@126. com

\section{ABSTRACT}

Objective To test whether intensive atorvastatin (ATV) increases the efficacy of transplantation with autologous bone marrow mononuclear cells (MNCs) in patients suffering from anterior ST-elevated myocardial infarction (STEMI).

Methods This clinical trial was under a $2 \times 2$ factorial design, enrolling 100 STEMI patients, randomly into four groups of regular (RA) or intensive ATV (IA) with MNCs or placebo. The primary endpoint was the change of left ventricular ejection fraction (LVEF) at 1-year follow-up from baseline, primarily assessed by MRI. The secondary endpoints included other parameters of cardiac function, remodelling and regeneration determined by MRI, echocardiography, positron emission tomography (PET) and biomarkers.

Results All the STEMI patients with transplantation of MNCs showed significantly increased LVEF change values than those with placebo $(p=0.01)$ with only in the IA+MNCs patients group demonstrating significantly elevation of LVEF than in the IA+placebo group $(+12.6 \%$ $(95 \% \mathrm{Cl} 10.4$ to 19.3$)$ vs $+5.0 \%(95 \% \mathrm{Cl} 4.0$ to 10.0$)$, $\mathrm{p}=0.001)$, pointing to a better synergy between ATV and MNCs $(p=0.019)$. PET analysis revealed significantly increased viable areas of myocardium $(p=0.015)$, while the scar sizes $(p=0.026)$ and blood aminoterminal pro-Btype natriuretic peptide $(p<0.034)$ reduced. All these above benefits of MNCs were also attributed to IA+MNCs instead of RA+MNCs group of patients with STEMI.

Conclusions Intensive ATV treatment augments the therapeutic efficacy of MNCs in patients with anterior STEMI at the convalescent stage. The treatment with the protocol of intensive ATV and MNC combination offers a clinically essential approach for myocardial infarction. Trial registration number NCT00979758.

\section{INTRODUCTION}

Many clinical trials have shown that transplantation of bone marrow cells may possess the therapeutic benefit for acute myocardial infarction (AMI) ${ }^{1-5}$ Mononuclear cell (MNC) transplantation reportedly promotes

\section{Key questions}

What is already known about this subject?

- Many clinical trials revealed the potential of bone marrow mononuclear cells (MNCs) therapy for acute myocardial infarction (AMI). However, the limited efficacy of MNCs was not as promising as those from the animal studies, which is the main bottle-neck limitation since the transplanted stem cells barely retain and hardly survive in the infarct area because of the harsh postinfarct microenvironments.

- For improving the effectiveness of stem cells therapy, we have reported that pretreatment with high dose of statins with anti-inflammation and pleiotropic effects on cardiovascular cells can increase survival of implanted MSCs in the infarcted myocardium, accompanied by improvement of left ventricular ejection fraction (LVEF) and myocardial metabolism in porcine AMI models.

myocardial repair. ${ }^{6}$ However, there is relatively limited improvement of left ventricular ejection fraction (LVEF), which is not as promising as those from the animal studies. ${ }^{89}$ The main bottle-neck limitations may be that few transplanted stem cells survive the harsh microenvironments of the infarcted cardiac tissue. $^{10}$

In order to improve the effectiveness of stem cell therapy, we have previously explored the possibility that preconditioning the ischaemic myocardium with high doses of statins exerts pleiotropic effects on cardiovascular cells. ${ }^{1011}$ Our previous studies in porcine AMI models have demonstrated that intensive statin administration increases the potency of implanted MSCs for survival, repair and regeneration in the infarcted myocardium, with improved LVEF and myocardial metabolism $^{11} 12$ through reducing the harshness of microenvironments which contain 


\section{What does this study add?}

- We hypothesised that pretreatment with statins may offer a novel strategy for improving the harsh microenvironment in infracted myocardium and augmenting the efficacy of MNCs transplantation in AMI patients.

- We conducted the trial with a $2 \times 2$ factorial design, and enrolled 100 patients with extensive anterior wall ST-elevated myocardial infarction, who were randomly allocated into four groups of regular (RA) or intensive atorvastatin (IA) with MNCs or placebo. The primary endpoint was the change value of LVEF at 1-year follow-up with MRI.

- The change value of LVEF in total MNCs patients was significantly increased compared with that in total placebo patients $(p=0.01)$, with only in IA+MNCs significantly enhanced compared with IA+placebo groups $(+12.6 \%(95 \% \mathrm{Cl} 10.4$ to 19.3$)$ vs $+5.0 \%(95 \% \mathrm{Cl}$ 4.0 to 10.0), $p=0.001$ ) and a significant synergy between atorvastatin and MNCs ( $p=0.019)$. Positron emission tomography revealed significant increase in the viable myocardial area $(p=0.015)$ with significant reductions in scar sizes $(p=0.026)$ of MRI and blood aminoterminal pro-B-type natriuretic peptide $(p<0.034)$. All these benefits in total MNCs patients occurred only in IA+MNCs group as well.

How might this impact on clinical practice?

- IA in peritransplant period remarkably augments the therapeutic efficacy of MNCs in patients with anterior AMI even at the convalescent stage, thus providing a novel strategy for enhancing the efficacy of MNC transplantation in clinical treatment of AMI patients.

inflammatory and cytotoxic substances, that is, 'fertilising the poor soil' at the infarcted region. Our recent work has also shown that the intensive statin treatment activates endothelial nitric oxide synthase. ${ }^{12}$ Interestingly, our previous study has shown that treatment with statins

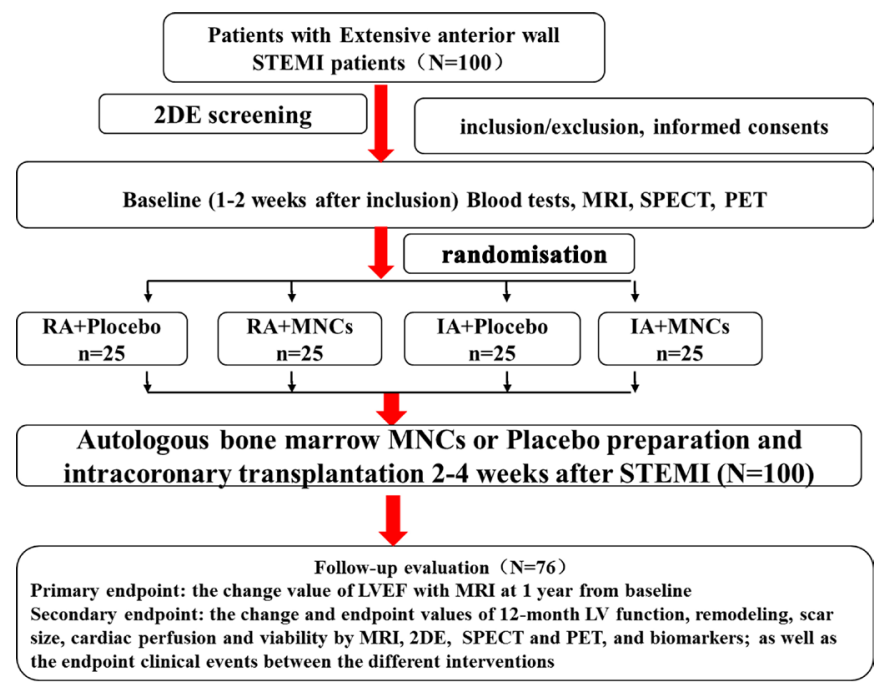

Figure 1 Schematic representation of the study design and grouping. 2DE, two-dimensional echocardiography; IA, intensive atorvastatin; LVEF, left ventricular ejection fraction; MNCs, mononuclear cells; PET, positron emission tomography; RA, routine atorvastatin; SPECT, single-photon emission CT; STEMI, ST-segment elevation myocardial infarction. promotes the cardiomyogenesis of stem cells via inducing expression of promyogenic genes. ${ }^{13}$

Therefore, we have pursued a clinical study to translate the preclinical research into the combined application of intensive statin and MNCs in patients with ST-elevated myocardial infarction (STEMI) to offer a novel strategy for improving the postinfarct cardiac microenvironment and augmenting the efficacy of MNC transplantation in AMI patients.

\section{METHODS \\ Participants}

A total of 100 STEMI patients were enrolled into a phase-II, randomised, double-blind, placebo-controlled trial in a single-centre between January 2009 and February 2014. The inclusion criteria were as follows: (1) ages between 30 and 80 years; (2) 2-4 weeks after the onset STEMI in the left ventricular anterior wall; (3) evidence of non-viable infarcts by positron emission tomography (PET) (4) LVEF $\leq 45 \%$ by two-dimensional echocardiography (2DE). The exclusion criteria are as follows: (1) non-STEMI (NSTEMI), (2) LVEF $>45 \%$, (3) severe valve heart disease or mechanical complications including ventricular septal perforation, tendon or papillary muscle rupture, (4) other systemic diseases including acute infectious diseases, haematologic diseases, severe kidney dysfunction, serious liver damage, unstable brain lesions, malignant tumours, pregnancy and severe mental or physical disability who will not be able to follow-up.

\section{Study design and procedures}

The trial was conducted following a $2 \times 2$ factorial design. By applying a web-based automated random number generator as previously described, ${ }^{11}{ }^{12}$ a treatment schedule will be prepared by a designated researcher who will have no contact with any participants, and patients at the convalescent stage (2-4 weeks post-STEMI) were randomly allocated to treatment with regular (RA, 20 $\mathrm{mg} /$ day) or intensive (IA, $80 \mathrm{mg} /$ day) atorvastatin (ATV, Lipitor, Pfizer Pharmaceutical Company) for 3 days, and then to intracoronary infusion with autologous bone marrow-derived MNCs or placebo. In essence, patients were divided into four groups (25 each) of regular atorvastatin with placebo (RA+placebo) or MNCs (RA $+\mathrm{MNCs}$ ), and intensive atorvastatin (IA) with placebo (IA+placebo) or MNCs (IA+MNCs). After MNCs transplantation, only patients in the two IA groups received 4 days more of IA then followed by regular atorvastatin as in the two RA groups with clinical followed-up and evaluation for up to 1 year. All the investigators, including clinical and imaging professionals, and the patients were blinded to the information of treatment and grouping. Furthermore, the tests and data processing of MRI, 2DE, PET with single-photon emission CT (SPECT) were, respectively, and independently performed by the designated doctors in the each core laboratory who were also blinded to the information (figure 1). 


\section{Primary and secondary endpoints}

The primary endpoint was the change value of LVEF from the baseline to the end of 1-year follow-up. The secondary endpoints included the change and endpoint values of other functional and morphological parameters and infarct scar sizes as measured by 2DE and MRI, myocardial perfusion and viability by SPECT and PET, and the cardiac biomarkers in the blood, including aminoterminal pro-B-type natriuretic peptide (NT-proBNP), high sensitivity $\mathrm{C}$ reactive protein (hs-CRP) and endothelin. Clinical events were monitored closely, including cardiac death, myocardial reinfarction, repeated revascularisation and malignant ventricular arrhythmias, such as ventricular tachycardia, flutter or fibrillation.

\section{Preparation and intracoronary infusion of autologous bone marrow MNCs}

Following the protocol reported previously by our team and others, ${ }^{11} 1214$ MNCs were prepared from the bone marrow aspirated from the posterior iliac crest under local anaesthesia. The preparation of MNCs or placebo in detail was described in the online supplementary data part 1. Patients who underwent the primary percutaneous coronary intervention were examined by coronary angiography and then MNCs or placebo infusion was performed. Otherwise, patients were first revascularised for the infarct-related coronary artery left anterior descending and then given by the MNCs infusion following the index procedure. The stem cell delivery in detail was described in the online supplementary data part 2.

\section{Cardiac MRI}

The MRI examination and analysis in detail were described in the online supplementary data part 3 .

\section{Two-dimensional echocardiography (2DE)}

The 2DE examination and analysis in detail were described in the online supplementary data part 4 .

\section{Analysis of myocardial perfusion and viability by SPECT and PET}

Myocardial perfusion was assessed by a dual-head SPECT (Siemens Medical, GER) $90 \mathrm{~min}$ after intravenously injecting a dose of $740 \mathrm{MBq}$ technetium-99m sestamibi (99mTc-MIBI), and PET scans were performed within 2 days after SPECT (the process of examination and analysis in detail were described in the online supplementary data part 5).

\section{Analysis of blood biomarkers}

The NT-proBNP and endothelin-1 were analysed using an immunoassay according to manufacturer's instructions (Roche Elecys, Roche Diagnostics, Basel, Switzerland; R\&D Systems, Minneapolis, MN, USA). The hs-CRP concentrations were measured with an automated chemistry analyser using commercial kits (Abbott Aeroset, Minnesota, USA).

\section{Safety monitoring}

All the participants were monitored for adverse events and the associated information would be transmitted to the Data and Safety Monitoring Board, the Institutional Review Board of our centre and China Food and Drug Administration (CFDA). The trial stopping rules were developed in consultation with the CFDA.

\section{Statistical analyses and sample size calculation}

The clinical trial was designed using a $2 \times 2$ factorial model in which intervention effectiveness was compared between patients treated with statin-enhanced and control MNCs. Patients with implantation of MNCs were compared when they received regular or intensive statin therapy. Such comparisons were performed in a multivariable regression model, including the interventions, corresponding interactions as well as the values of outcome variables. Power analysis was performed to determine the sample sizes with the statistical significance level taken as one-sided 0.025 and the power as $80 \%$. The quantitative data were presented as mean $\pm \mathrm{SD}$. Categorical data were presented as frequencies and percentages. Continuous variables at baseline with those at follow-up were compared with the paired analysis of variance. Continuous variables with a non-normal distribution between groups were compared using the Wilcoxon rank sum test and the Mann-Whitney test. Statistical significance was set at $\mathrm{p}<0.05$. Data were analysed using SPSS V.19.0 (online supplementary data part 6 ).

\section{RESULTS}

All the 100 participants enrolled in this study received the baseline assessments. Among them, 76 patients did completion of the 1-year end-point follow-up assessments and their data entered the final analysis. The remaining 24 participants who did not complete the 1-year endpoint evaluations due to geological distance and personal inconvenience were excluded from final analysis. Comparison in the baseline characteristics between the patients with and without completion of final assessments indicated no significant difference (online supplementary table 1). In addition, no significant differences in the baseline characteristics and all the parameters of coronary lesions and interventions occurred between the four groups or under RA and IA groups (table 1 and online supplementary table 2).

The analysis of the blood lipid profile parameters from baseline to endpoint showed that the decrease in LDL-C levels was significantly more only in IA treatment patients than that in RA patients $(-0.78 \mathrm{mmol} / \mathrm{L}(-0.97$ to -0.59$)$ vs $-0.32 \mathrm{mmol} / \mathrm{L}(-0.51$ to -0.18$), \mathrm{p}=0.013$ ) (online supplementary table 3$)$.

\section{MRI assessment of cardiac performance, remodelling and scar sizes}

As shown in figure 2 and table 2A,B the change value of LVEF from baseline to endpoint in total MNCs group was 
Table 1 Baseline characteristics of the four groups

\begin{tabular}{|c|c|c|c|c|c|}
\hline & $\begin{array}{l}\text { RA+placebo } \\
(n=19)\end{array}$ & $\begin{array}{l}\text { RA+MNCs } \\
(n=20)\end{array}$ & $\begin{array}{l}\text { IA+placebo } \\
(n=17)\end{array}$ & $\begin{array}{l}\text { IA+MNCs } \\
(n=20)\end{array}$ & $P$ value \\
\hline Male (\%) & $18(94.7)$ & $18(90.0)$ & $15(88.2)$ & $17(85.0)$ & 0.797 \\
\hline Age (years) & $51.7 \pm 9.1$ & $57.0 \pm 12.7$ & $50.1 \pm 13.0$ & $52.9 \pm 13.8$ & 0.360 \\
\hline BMI $\left(\mathrm{kg} / \mathrm{m}^{2}\right)$ & $25.5 \pm 6.2$ & $25.2 \pm 3.0$ & $26.6 \pm 4.6$ & $26.2 \pm 3.0$ & 0.773 \\
\hline Killip class & $1.89 \pm 1.29$ & $1.60 \pm 0.88$ & $1.53 \pm 0.87$ & $1.75 \pm 1.07$ & 0.720 \\
\hline $\operatorname{IABP}(\%)$ & $3(15.8)$ & $1(5.0)$ & $1(5.9)$ & $4(20.0)$ & 0.386 \\
\hline Hypertension (\%) & $10(52.6)$ & $12(60.0)$ & $7(41.2)$ & $9(45)$ & 0.668 \\
\hline Diabetes (\%) & $6(31.6)$ & $4(20.0)$ & $4(23.5)$ & $3(15.0)$ & 0.666 \\
\hline Hyperlipidaemia (\%) & $8(42.1)$ & $3(15.0)$ & $8(47.1)$ & $7(35.0)$ & 0.168 \\
\hline Stroke $(\%)$ & 0 & 0 & 0 & $1(5.0 \%)$ & 0.417 \\
\hline Smoking (\%) & $17(89.5)$ & $13(65.0)$ & $13(76.5)$ & $10(50.0)$ & 0.050 \\
\hline CHD family history (\%) & $4(21.1)$ & $2(10.0)$ & $3(17.6)$ & $2(10.0)$ & 0.729 \\
\hline Previous Ml (\%) & $2(10.5)$ & $4(20 \%)$ & $0(0)$ & $0(0)$ & 0.055 \\
\hline Previous coronary revascularisation (\%) & $1(5.3)$ & $2(10.0)$ & $0(0)$ & $0(0)$ & 0.503 \\
\hline $\mathrm{SBP}(\mathrm{mm} \mathrm{Hg})$ & $113.1 \pm 16.0$ & $110.7 \pm 13.0$ & $120.8 \pm 17.6$ & $115.2 \pm 19.2$ & 0.307 \\
\hline HR (bpm) & $80.6 \pm 19.2$ & $75.8 \pm 14.8$ & $76.3 \pm 10.9$ & $82.1 \pm 16.9$ & 0.520 \\
\hline ALT (IU/L) & $86.4 \pm 76.1$ & $57.0 \pm 41.9$ & $46.2 \pm 41.2$ & $56.4 \pm 32.8$ & 0.100 \\
\hline Scr (umol/L) & $84.2 \pm 10.9$ & $89.0 \pm 19.7$ & $87.5 \pm 17.0$ & $85.9 \pm 14.7$ & 0.804 \\
\hline HbA1c (\%) & $6.4 \pm 1.2$ & $6.5 \pm 1.4$ & $6.6 \pm 1.5$ & $6.2 \pm 1.3$ & 0.866 \\
\hline WBC $\left({ }^{*} 10^{9} / L\right)$ & $9.9 \pm 3.5$ & $9.8 \pm 5.5$ & $8.8 \pm 2.4$ & $9.0 \pm 4.3$ & 0.821 \\
\hline HCT (\%) & $42.3 \pm 5.0$ & $43.3 \pm 4.5$ & $39.9 \pm 3.7$ & $39.5 \pm 8.9$ & 0.155 \\
\hline $\mathrm{HGB}(\mathrm{g} / \mathrm{L})$ & $140.1 \pm 19.3$ & $145.6 \pm 16.3$ & $138.3 \pm 10.0$ & $136.6 \pm 14.1$ & 0.295 \\
\hline PLT ( $\left.{ }^{*} 10^{9} / \mathrm{L}\right)$ & $262.8 \pm 119.9$ & $226.3 \pm 92.1$ & $258.7 \pm 93.0$ & $252.4 \pm 83.0$ & 0.656 \\
\hline \multicolumn{6}{|l|}{ Medications } \\
\hline Aspirin (\%) & $19(100)$ & $20(100)$ & $17(100)$ & $20(100)$ & NS \\
\hline Clopidogrel (\%) & $19(100)$ & $20(100)$ & $17(100)$ & $20(100)$ & NS \\
\hline Beta-blockers (\%) & $17(89.5)$ & $19(95.0)$ & $16(94.1)$ & $18(90.0)$ & 0.912 \\
\hline RAASi (\%) & $14(73.7)$ & $15(75.0)$ & $13(76.5)$ & $14(70.0)$ & 0.844 \\
\hline Spirolactone (\%) & $13(68.4)$ & $12(60.0)$ & $11(64.7)$ & $14(70.0)$ & 0.638 \\
\hline Diuretics (\%) & $15(78.9)$ & $14(70.0)$ & $13(76.5)$ & $13(65.0)$ & 0.597 \\
\hline
\end{tabular}

ALT, alanine aminotransferase; BMI, body mass index; CHD, coronary heart disease; HbA1c, glycosylated haemoglobin; HCT, haematocrit; HGB, haemoglobin; HR, heart rate; IA, intensive aotrvastain; IABP, intra-aortic balloon pump; LDL-C, low-density lipoprotein cholesterol; MI, myocardial infarction; MNCs, mononuclear cells; NS, not significant; PLT, platelet; RA, regular atorvastatin; RAASi, renin angiotensin aldosterone system inhibitor; SBP, systolic blood pressure; Scr, serum creatinine; WBC, white blood cell.

significantly increased compared with that in total placebo group $(+10.0 \%$ (95\% CI 8.0 to 12.6$)$ vs $+5.0 \%$ (95\% CI 3.5 to 9.2$), \mathrm{p}=0.010)$. However, the significant changes were observed only between IA intragroups $(+12.6 \%$ (95\% CI 10.4 to 19.3 ) vs $+5.0 \%$ (95\% CI 4.0 to 10.0$), \mathrm{p}=0.001$ ) but not between RA intragroups $(\mathrm{p}=0.809)$. There was a significantly synergistic improvement in LVEF between atorvastatin and MNCs $(p=0.019)$. The endpoint value of LVEF in total MNCs group was also significantly increased compared with that in total placebo group $(46.0 \%(95 \%$ CI 44.0 to 49.5 ) vs $39.5 \%$ (95\% CI 37.0 to 43.3 ), $\mathrm{p}=0.009$ ), with only in IA+MNCs being significantly higher than in IA+placebo groups as well $(47.5 \%$ (95\% CI 45.0 to 52.6$)$ vs $38.0 \%$ (95\% CI 34.0 to 41.0 ), $\mathrm{p}=0.001$ ).
Similarly, the MNCs implantation significantly reduced the scar sizes of MRI compared with placebo $(p=0.010)$, with only in patients receiving intensive instead of regular atorvastatin reaching significance, too $(\mathrm{p}=0.026)$. No synergistic effect was detected between intensive ATV and MNCs therapy ( $\mathrm{p}=0.572$ ).

In order to evaluate the impacts of the missing data (lost to follow-up), we also added a sensitivity analysis on the primary endpoint (changes on LVEF between baseline and 1-year follow-up) according to the intention-to-treat principle. Last observation carried forward strategy had been used to impute the missing data of LVEF. Because the 24 patients did not have any LVEF data measured with MRI during their follow-up period, the baseline values 


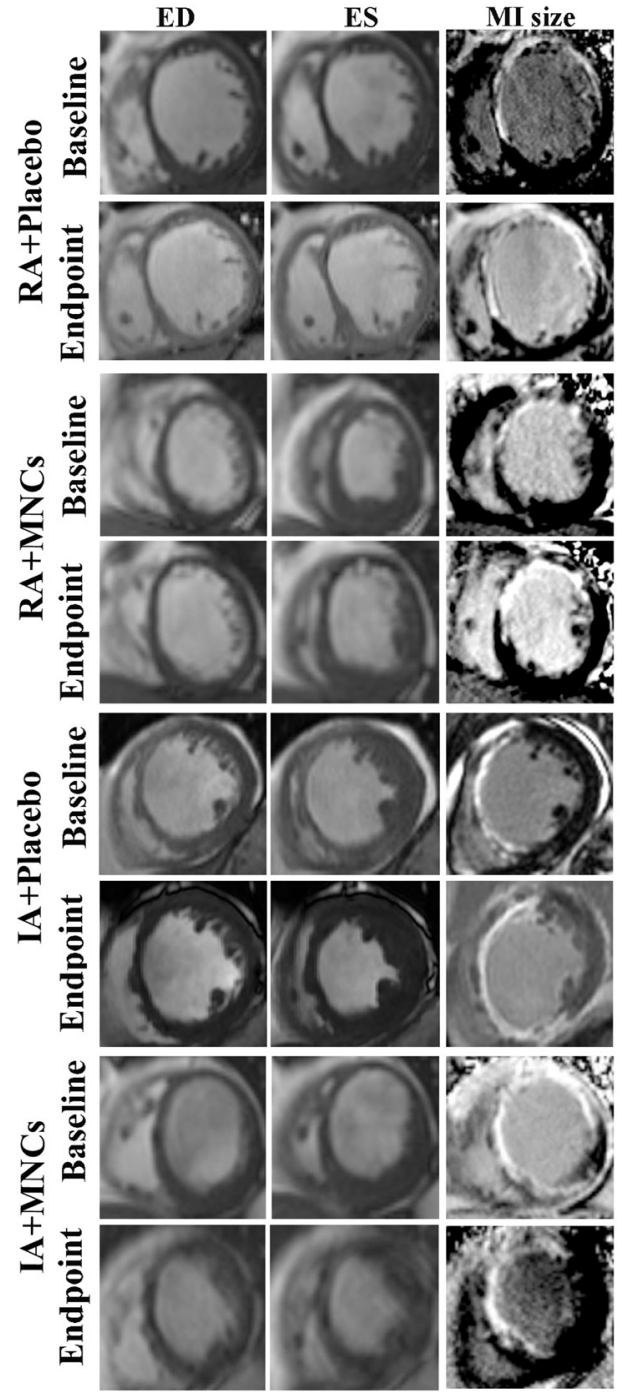

Figure 2 Representative MRI images of cardiac left ventricle in myocardial infarct (MI) patients treated with intensive (IA) or regular atorvastatin (RA) in combination with or without transplantation of mononuclear cells (MNCs). MRI was conducted at short axis in the end-diastolic (ED) or endsystolic (ES) phase.

were used for the imputation. After the 'missing LVEF values' of the 24 patients were added in the final analyses, the results were consistent with those achieved from only 76 patients who completed the endpoint examinations (online supplementary table 4 ).

\section{Analysis of cardiac function and remodelling by echocardiography}

As shown in online supplementary figure 1 and online supplementary table 5, as with MRI evaluation, 2DE demonstrated significant change values of LVEF from baseline to endpoint in total MNCs group and only in IA+MNCs group than total placebo group $(+9 \%(5,10)$ vs $+4 \%(2,6) ; \mathrm{p}=0.022)$ and IA+placebo group $(+10 \%(8,12)$ vs $+4 \%(0,10) ; \mathrm{p}=0.045)$, with no significant synergy between atorvastatin treatment and $\mathrm{MNC}$ implantation $(\mathrm{p}=0.594)$.
Consistently, the change values of both EDV and ESV significantly decreased in total MNCs group compared with those in total placebo group ( $\mathrm{p}=0.041$ and $\mathrm{p}=0.03$ ), with both of the change values being significantly reduced only in IA+MNCs compared with IA+placebo groups $(\mathrm{p}=0.014$ and $\mathrm{p}=0.020)$, with no significant synergy between atorvastatin and MNCs $(\mathrm{p}=0.217$ and $\mathrm{p}=0.444)$.

\section{Assessment of myocardial perfusion and viability by SPECT and PET}

The change values of both perfusion defect sizes and summed rest score were not significantly different between the total MNCs and placebo groups, or between IA and RA groups (online supplementary table 6). However, the change value of FDG score in total MNCs were significantly increased compared with that in total placebo groups $(p=0.017)$, and this benefit was observed only in IA+MNCs compared with IA+placebo group $(\mathrm{p}=0.015)$, indicating possible myocardial regeneration occurred in the IA+MNCs patients. No synergistic effect was observed between atorvastatin and MNCs though ( $\mathrm{p}=0.081)$ (figure 3)

\section{Changes in blood biomarkers}

The change values of blood NT-proBNP from baseline to endpoint in the total MNCs patients were significantly higher than that in total placebo patients $(p=0.023)$, with both change and endpoint values significantly increased and decreased respectively, only between IA subgroups $(\mathrm{p}=0.034$ and $\mathrm{p}=0.005$, respectively), indicating that IA treatment improved the left ventricular function evaluated with specific biomarkers as well. There were no significant synergy between atorvastatin treatment and MNCs therapy $(\mathrm{p}=0.165)$. Meanwhile, the endpoint value of hs-CRP was significantly lower only in total IA than RA groups $(p=0.001)$, and the change value of endothelin was significantly higher only in IA+MNCs than in IA+placebo groups as well $(\mathrm{p}=0.046)$ (figure 4 and online supplementary table 7 ).

\section{Assessment of safety}

The safety of intracoronary MNCs infusion was evidenced by the following observations throughout the treatment and follow-up period: (i) no death or recurrent MI in patients receiving treatments; (ii) no target lesion revascularisation; no new arrhythmia, for example, sustained ventricular tachycardia or fibrillation. All the enrolled patients including 24 patients who did not finish the endpoint instrumental examinations completed telephone follow-up survey, and they all were alive to the moment of current report (online supplementary table $8)$.

\section{DISCUSSION}

The present study provides the first clinical evidence that intensive statins apparently improves the therapeutic effects of MNCs transplantation in terms of LVEF elevation for patients with extensive anterior STEMI at 
Table 2 (A) Left ventricular function, remodelling and scar size assessment by MRI. (B) Comparison of MRI parameters between ATV groups

\begin{tabular}{|c|c|c|c|c|}
\hline A & $\begin{array}{l}\text { Total RA } \\
(n=39)\end{array}$ & $\begin{array}{l}\text { Total IA } \\
(\mathrm{n}=37)\end{array}$ & $\begin{array}{l}\text { Total placebo } \\
(\mathrm{n}=36)\end{array}$ & $\begin{array}{l}\text { Total MNCs } \\
(n=40)\end{array}$ \\
\hline \multicolumn{5}{|l|}{ LVEF } \\
\hline Baseline & $36.0(33.0,39.4)$ & $32.0(31.0,34.4)$ & $33.5(31.5,35.0)$ & $34.2(32.7,36.5)$ \\
\hline Endpoint & $44.0(41.0,46.5)$ & $43.0(39.0,46.0)$ & $39.5(37.0,43.3)$ & $46.0(44.0,49.5)^{\star}$ \\
\hline Adjusted difference $\left.{ }^{(95 \%} \mathrm{Cl}\right)$ & $6.9(4.0$ to 9.0$)$ & $10.0(7.6$ to 12.1$)$ & 5.0 (3.5 to 9.2 ) & $10.0(8.0$ to 12.6$)$ \\
\hline$P$ value & 0.089 & & 0.01 & \\
\hline \multicolumn{5}{|l|}{$\operatorname{EDVi}\left(\mathrm{mL} / \mathrm{m}^{2}\right)$} \\
\hline Baseline & $81.5(74.4,89.7)$ & $82.2(75.0,91.0)$ & $81.9(74.4,89.7)$ & $81.8(73.2,90.0)$ \\
\hline Endpoint & $67.9(62.0,75.6)$ & $71.0(63.7,81.9)$ & $72.3(62.5,78.1)$ & $68.5(64.6,78.3)$ \\
\hline Adjusted difference $\dagger(95 \% \mathrm{Cl})$ & $-7.8(-18.8$ to -3.0$)$ & $-21.4(-24.4$ to -3.2$)$ & $-6.9(-17.1$ to -1.7$)$ & $-9.2(-18.0$ to -0.9$)$ \\
\hline$P$ value & 0.718 & & 0.832 & \\
\hline \multicolumn{5}{|l|}{ ESVi $\left(\mathrm{mL} / \mathrm{m}^{2}\right)$} \\
\hline Baseline & $49.8(46.6,61.0)$ & $55.7(51.0,61.0)$ & $50.4(47.0,60.5)$ & $55.6(49.3,62.4)$ \\
\hline Endpoint & $39.0(32.0,44.0)$ & $37.0(32.0,45.0)$ & $37.0(32.5,45.0)$ & $39.0(32.0,44.2)$ \\
\hline Adjusted difference $\dagger(95 \% \mathrm{Cl})$ & $-9.7(-12.2$ to -0.8$)$ & $-11.2(-29.9$ to -0.7$)$ & $-8.1(-21.4$ to -1.2$)$ & $-18.5(-26.3$ to -6.1$)$ \\
\hline$P$ value & 0.533 & & 0.234 & \\
\hline \multicolumn{5}{|l|}{ Scar size (mm3) } \\
\hline Baseline & $39.2(31.8,42.0)$ & $41.5(33.0,44.8)$ & $39.2(32.4,43.9)$ & $40.0(32.7,42.2)$ \\
\hline Endpoint & $40.2(35.7,44.5)$ & $35.9(33.0,43.8)$ & $38.6(34.6,50.1)$ & $36 \cdot 5(31 \cdot 0,42.0) \ddagger$ \\
\hline Adjusted difference $(95 \% \mathrm{Cl})$ & $1.67(-0.16$ to 4.80$)$ & 0.81 (-3.32 to 2.62) & 2.60 (1.18 to 6.94$)$ & $-2.65(-5.10$ to 1.60$)$ \\
\hline \multirow[t]{2}{*}{$P$ value } & \multicolumn{2}{|l|}{0.659} & \multicolumn{2}{|l|}{0.01} \\
\hline & Total RA ( $n=39)$ & & Total IA $(n=37)$ & \\
\hline B & RA+placebo $(n=19)$ & RA+MNCs $(n=20)$ & IA+placebo $(n=17)$ & IA+MNCs $(n=20)$ \\
\hline \multicolumn{5}{|l|}{ LVEF } \\
\hline Baseline & $37.8(29.7,40.7)$ & $35.5(33.0,38.8)$ & $32.5(30.4,34.7)$ & $31.6(29.0,36.0)$ \\
\hline Endpoint & $43.6(37.0,46.5)$ & $45.5(41.1,49.5)$ & $38.0(34.0,41.0)$ & $47.5(45.0,52.6) \S$ \\
\hline Adjusted difference $†(95 \% \mathrm{Cl})$ & 3.4 (0.3 to 12.6$)$ & $7.8(6.3$ to 10.0$)$ & $5.0(4.0$ to 10.0$)$ & 12.6 (10.4 to 19.3$)$ \\
\hline$P$ value & 0.809 & & 0.001 & \\
\hline \multicolumn{5}{|l|}{$\operatorname{EDVi}\left(\mathrm{mL} / \mathrm{m}^{2}\right)$} \\
\hline Baseline & $81.5(74.4,92.1)$ & $82.5(67.5,92.7)$ & $82.2(73.5,101.0)$ & $81.8(73.2,92.6)$ \\
\hline Endpoint & $71.8(61.1,75.6)$ & $67.1(61.0,79.4)$ & $76.0(59.6,94.0)$ & $70.3(65.0,81.9)$ \\
\hline Adjusted difference $+(95 \% \mathrm{Cl})$ & $-3.3(-13.2$ to 0.8$)$ & $-11.5(-25.4$ to -2.4$)$ & $-8.3(-18.6$ to 4.4$)$ & $-8.4(-16.4$ to -0.19$)$ \\
\hline$P$ value & 0.799 & & 0.987 & \\
\hline \multicolumn{5}{|l|}{ ESVi $\left(\mathrm{mL} / \mathrm{m}^{2}\right)$} \\
\hline Baseline & $49.1(45.0,63.0)$ & $52.9(41.0,65.5)$ & $54.1(43.7,61.0)$ & $56.0(49.5,67.4)$ \\
\hline Endpoint & $36.5(31.0,47.0)$ & $39.1(29.5,44.1)$ & $37.0(29.2,61.0)$ & $38.0(30.2,47.9)$ \\
\hline Adjusted difference $\dagger(95 \% \mathrm{Cl})$ & $-4.5(-8.5$ to 0.7$)$ & $-17.5(-27.8$ to -6.1$)$ & $-21.4(-24.3$ to 1.4$)$ & $-21.1(-30.1$ to -2.0$)$ \\
\hline$P$ value & 0.556 & & 0.290 & \\
\hline \multicolumn{5}{|l|}{ Scar size $\left(\mathrm{mm}^{3}\right)$} \\
\hline Baseline & $39.3(30.5,47.9)$ & $39.1(30.3,45.4)$ & $33.8(31.8,47.8)$ & $41.6(32.3,49.7)$ \\
\hline Endpoint & $40.2(33.0,51.1)$ & $40.0(31.4,43.7)$ & $36.8(33.8,55.4)$ & $34.6(28.0,41.2)$ \\
\hline Adjusted difference $+(95 \% \mathrm{Cl})$ & 2.06 (0.64 to 8.13 ) & $-1.18(-4.64$ to 3.77$)$ & 3.06 (0.81 to 11.59$)$ & $-3.13(-5.63$ to 1.13$)$ \\
\hline$P$ value & 0.161 & & 0.026 & \\
\hline
\end{tabular}

*Endpoint values in total MNCs group vs total placebo group $(p=0.009)$.

†Values are median $(95 \% \mathrm{Cl})$, and adjusted for values at baseline and the other intervention.

$\ddagger$ Endpoint values in total MNCs group vs total placebo group $(p=0.008)$.

$\S$ The comparison of endpoint values in $I A+M N C$ s group vs $I A+$ placebo group $(p=0.001)$.

ATV, atorvastatin; EDVi, end-diastolic volume index; ESVi, end-systolic volume index; IA, intensive atorvastatin; LVEF, left ventricular ejection fraction; MNCs, mononuclear cells; RA, regular atorvastatin. 


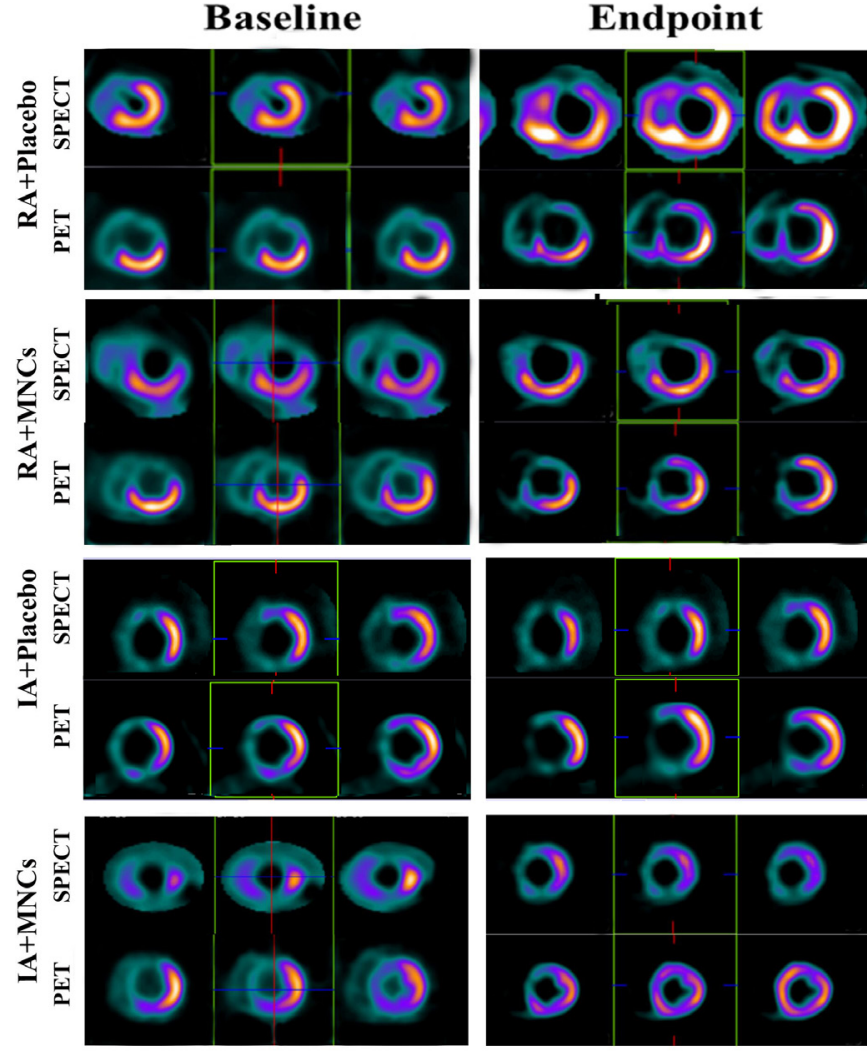

Figure 3 The representative images of SPECT and PET denoting myocardial regeneration after combined treatment with intensive atorvastatin (IA) and MNCs in the same patient shown in figure 2. A, intensive atorvastatin; MNCs, mononuclear cells; PET, positron emission tomography; RA, regular atorvastatin; SPECT, single-photon emission CT

the convalescent stage. The main benefits included (1) enhancement of cardiac performance with LVEF value increasing by $10 \%$ or even higher; (2) attenuation of LV remodelling; (3) decrease of scar size with the increase in the area of myocardial viability; (4) reduction in blood NT-pro BNP levels.

With a mixture of multiple types of cells, the bone marrow MNCs provide the ecosystem that comprises various cell lineages, including haematopoietic progenitor cells, endothelial progenitor cells, stromal cells and others, bearing characteristics of plasticity and availability. ${ }^{14}$ The positive results from several early clinical trials have shown that intracoronary infusion of bone marrow MNCs in AMI patients has therapeutic effects, such as the left ventricular function improvement and attenuation of negative remodelling. ${ }^{1516}$ However, the reported efficacy is very low with LVEF elevation by only $2.5 \%-3.9 \%,{ }^{67}$ and even negative in later clinical trials. ${ }^{9} 1718$ The low rates of survival of implanted MNCs in infarct area are considered to be the main factors to limit the effectiveness of the stem cell therapy because of bad microenvironment in the infarct myocardium. The present clinical trial is the first to demonstrate that pretreatment with intensive oral statin markedly augments the efficacy of MNCs therapy with LVEF elevating by $10 \%$ or even more, since it improves the harsh microenvironment of infarct region as like 'fertilisation of barren land'. Our observations suggest that intensive ATV preconditions is the prerequisite in stem cell therapy. This benefit provided with the combination therapy of intensive statin and MNCs is much more than that from previous clinical studies of MNCs alone. ${ }^{711} 12$ The synergistic effects between intensive ATV and MNCs shown in the clinical trial are consistent with our previous reports in the porcine model in vivo as well. ${ }^{101219}$ To the best of our knowledge, this is the first clinical report that the combination protocol of bone marrow MNCs with intensive ATV has achieved such an impressive efficacy in patients with STEMI, denoting the essential role of 'fertilisation' with intensive ATV in clinical stem cell therapy.

The synergism between intensive ATV and MNC transplantation is supported by several cellular and molecular biomarkers in the hearts. Apart from the antiinflammatory and antiapoptotic effects by MSCs themselves, the intensive ATV treatment likely improves the microenvironment via the pleiotropic effects, such as the endothelial protection, anti-inflammation, antioxidative stress and antiapoptosis for 'fertilising the poor soil' in infarct area ${ }^{11121920}$ and then enhances the survival and angiogenesis of implanted MNCs in addition to
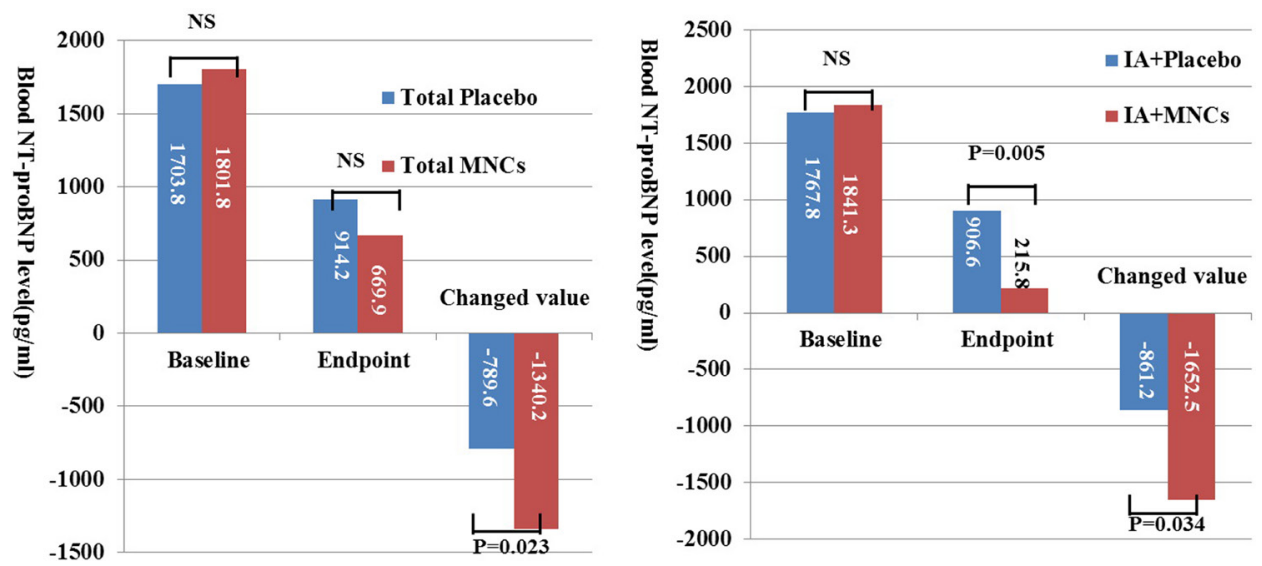

Figure 4 Changes in the blood levels of NT-proBNP after MNC transplantation with or without intensive ATV (IA). ATV, atorvastatin; MNCs, mononuclear cells; proBNP, pro-B-type natriuretic peptide; NS, not significant. 
upregulating of stromal derived factor-1 in infarct myocardial for MSCs directional homing. ${ }^{21-25}$ The combined treatment with intensive statin and MNCs reduces the scar size, which is also consistently with the results from recent animal studies reported by our team..$^{25} 26$

Moreover, the 2DE data obtained in the present study have shown that the intensive but regular ATV combined with MNC therapy can also reduce EDV and ESV, supporting an attenuation of left ventricular remodelling. This is consistent with those reported previously in other clinical studies. ${ }^{1-5}$ The mechanism for the ventricular remodelling attenuation with intensive instead of regular dose of atorvastatin remains unclear. Probably its stronger pleiotropic effects, including the lipid lowering and antiapoptotic action, contribute to the improvement.

Another interesting finding of this clinical trial is that the intensive but regular ATV combined with MNCs therapy can increase the area of viable myocardium at infarct region with reduction in scar size and blood level of heart failure biomarker NT-Pro BNP, suggesting the possibility of cardiac tissue repair and regeneration. It is consistent with our previous reports of animal experiments, ${ }^{10} 1219$ though has not been reported by the other clinical trial on AMI patients receiving MNCs. ${ }^{27}$ The myocardial regeneration in patients received the combined therapy with intensive ATV and MNCs transplantation may be, at least in part, attributed to activation and mobilisation of endogenous stem cells in the periinfarct regions by MSCs. ${ }^{28}$ However, more vigorous clinical evaluation is needed to obtain further evidence.

The optimal timing for stem cell transplantation remains a controversial issue. The majority of clinical trials with MNCs have applied the cell transplantation during the early period of time (usually within 7 days) following AMI, as reported in the BOOST and REPAIR-AMI trials. ${ }^{24}$ The natural expression of SDF-1 in infarct myocardium increases rapidly in 1 to 3 days post-AMI, and decline to the normal level within 1 week. ${ }^{29}$ From a clinical point of view, however, the early intracoronary infusion of MNCs for the critically ill patients is risky due to very low LVEF, dedicated unstable haemodynamics and complications of concomitant heart failure, etc. In the above dilemmatic situations, our current study choose delayed time points (average 4 weeks after STEMI) in intracoronary infusion of MNCs for the patients' safety. MNCs implantation at the convalescent phase of STEMI in this study results in an improvement of the efficacy with the protocol combined intensive ATV with MNC therapy, which is consistent with the preclinical data, ${ }^{30}$ though to some degrees, inconsistent with the majority of clinical trials. ${ }^{69}$ The underlying mechanisms are still unknown, possibly due to the composite effectiveness which includes the above-mentioned synergistic effects, the subsided harsh microenvironment of inflammation, and the remaining SDF-1 levels in the infarct myocardium, the increased CXCR4 expressions in endothelial progenitor cells, ${ }^{20}$ and the target migration of MSCs via the activating SDF-1/ CXCR4 axis $^{23}{ }^{24}$ by intensive statins.
In conclusion, the pretreatment with IA augments the efficacy of MNC transplantation in enhancing the cardiac performance, attenuating ventricular remodelling for patients with STEMI at the convalescent stage, probably through improving the harsh microenvironment in infarct zone and increasing implanted MNC survival and possible regeneration, revealing its essential role in clinical stem cell therapy.

\section{Author affiliations}

${ }^{1}$ Department of Cardiology, Center for Coronary Heart Disease, State Key Laboratory of Cardiovascular Disease, Fuwai Hospital, National Center for Cardiovascular Diseases, Chinese Academy of Medical Sciences and Peking Union Medical College, Beijing, China

${ }^{2}$ The Center for Cardiovascular Biology and Atherosclerosis, Department of Internal Medicine, University of Texas McGovern School of Medicine at Houston, Houston, Texas, USA

${ }^{3}$ Department of Cardiology, Beijing Chaoyang Hospital, Capital Medical University, Beijing, China

${ }^{4}$ Center for Cardiac Critical Care, Beijing Anzhen Hospital, Capital Medical University, Beijing Institute of Heart Lung and Blood Vessel Diseases, Beijing, China ${ }^{5}$ Department of Cardiology, Beijing Anzhen Hospital, Capital Medical University, Beijing Institute of Heart, Lung and Blood Vessel Diseases, Beijing, China ${ }^{6}$ Department of Nuclear Medicine, Fuwai Hospital, National Center for Cardiovascular Diseases, Chinese Academy of Medical Sciences and Peking Union Medical College, Beijing, China

${ }^{7}$ Department of Magnetic Resonance Imaging, Fuwai Hospital, National Center for Cardiovascular Diseases, Chinese Academy of Medical Sciences and Peking Union Medical College, Beijing, China

${ }^{8}$ Department of Echocardiography, State Key Laboratory of Cardiovascular Disease, Fuwai Hospital, National Center for Cardiovascular Diseases, Chinese Academy of Medical Sciences and Peking Union Medical College, Beijing, China

Acknowledgements We appreciate Dr Xiang-Feng Lu and Dr Yang Wang for their contributions to revision of the statistical methodology.

Contributors The coauthors including Y-JY, Y-JG, H-YQ and R-LG contributed to the planning, the coauthors including LS, NL, HW, X-QT, JH, P-SH, JX, RS, M-JL, S-HZ, W-CW and JQ contributed to the conduct and the coauthors including YW, $J Z, J-Y X$ and $Y-Y X$ contributed to the reporting of the work in the article. Among the above coauthors, professors $Y-J Y$ and $\mathrm{H}-\mathrm{YQ}$ are responsible for the overall contents as guarantors. We did not include any figure or table from another publication.

Funding Y-JY was supported by grants from CAMS Innovation Fund for Medical Sciences (CIFMS, 2016-12 M-1-009), National Basic Research Program (973 Program) in China (no: 2012CB518602, Ministry of Science and Technology), National High Technology Research and Development Program (863 Program) in China (no: 2013AA020101, Ministry of Science and Technology), National Natural Science Foundation of China (no: 81170129), Research Fund of Capital Medical Development (no: 2007-2018) and China Health and Medical Development Foundation (2008zhfj2, 2011-zhfj1, 2015-zhfj2). H-YQ was supported by grants from the Clinical and Translational Medicine Research Foundation of Chinese Academy of Medical Sciences (2019XK320061), and National Natural Science Foundation of China (nos: 81000091, 81670337).

\section{Competing interests None declared.}

Patient consent for publication Not required.

Ethics approval The institutional review boards of Fuwai Cardiovascular Hospital of the Chinese Academy of Medical Sciences reviewed and approved the study protocol.

Provenance and peer review Not commissioned; externally peer reviewed.

Data availability statement Data are available upon reasonable request. The related data can be requested from the co-author Professor $\mathrm{H}-\mathrm{YQ}$ via email at ahqhy712@163.com.

Open access This is an open access article distributed in accordance with the Creative Commons Attribution 4.0 Unported (CC BY 4.0) license, which permits others to copy, redistribute, remix, transform and build upon this work for any purpose, provided the original work is properly cited, a link to the licence is given, 
and indication of whether changes were made. See: https://creativecommons.org/ licenses/by/4.0/.

ORCID iD

Yue-Jin Yang http://orcid.org/0000-0002-1309-2667

\section{REFERENCES}

1 Strauer BE, Brehm M, Zeus T, et al. Repair of infarcted myocardium by autologous intracoronary mononuclear bone marrow cell transplantation in humans. Circulation 2002;106:1913-8.

2 Meyer GP, Wollert KC, Lotz J, et al. Intracoronary bone marrOw cell transfer after myocardial infarction: eighteen months' follow-up data from the randomized, controlled boost (bone marrOw transfer to enhance ST-elevation infarct regeneration) trial. Circulation 2006;113:1287-94.

3 Janssens S, Dubois C, Bogaert J, et al. Autologous bone marrowderived stem-cell transfer in patients with ST-segment elevation myocardial infarction: double-blind, randomised controlled trial. Lancet 2006:367:113-21.

4 Schächinger V, Erbs S, Elsässer A, et al. Intracoronary bone marrowderived progenitor cells in acute myocardial infarction. N Engl J Med 2006;355:1210-21.

5 Lunde K, Solheim S, Forfang K, et al. Anterior myocardial infarction with acute percutaneous coronary intervention and intracoronary injection of autologous mononuclear bone marrow cells: safety, clinical outcome, and serial changes in left ventricular function during 12-months' follow-up. J Am Coll Cardiol 2008;51:674-6.

6 Delewi R, Hirsch A, Tijssen JG, et al. Impact of intracoronary bone marrow cell therapy on left ventricular function in the setting of ST-segment elevation myocardial infarction: a collaborative metaanalysis. Eur Heart J 2014;35:989-98.

7 Jeevanantham V, Butler M, Saad A, et al. Adult bone marrow cell therapy improves survival and induces long-term improvement in cardiac parameters: a systematic review and meta-analysis. Circulation 2012;126:551-68.

8 Lunde K, Solheim S, Aakhus S, et al. Intracoronary injection of mononuclear bone marrow cells in acute myocardial infarction. $N$ Engl J Med 2006;355:1199-209.

9 Traverse JH, Henry TD, Ellis SG, et al. Effect of intracoronary delivery of autologous bone marrow mononuclear cells 2 to 3 weeks following acute myocardial infarction on left ventricular function: the LateTIME randomized trial. JAMA 2011;306:2110-9.

10 Ranganath SH, Levy O, Inamdar MS, et al. Harnessing the mesenchymal stem cell secretome for the treatment of cardiovascular disease. Cell Stem Cell 2012;10:244-58

11 Yang YJ, Qian HY, Huang J, et al. Atorvastatin treatment improved post-infarction microenvironments to facilitate the survivals and activities of implanted mesenchymal stem cells in swine hearts with acute myocardial infarction and reperfusion. Eur Heart $J$ 2008;29:1578-90.

12 Song L, Yang Y-J, Dong Q-T, et al. Atorvastatin enhance efficacy of mesenchymal stem cells treatment for swine myocardial infarction via activation of nitric oxide synthase. PLoS One 2013;8:e65702.

13 Yang C, Madonna R, Li Y, et al. Simvastatin-enhanced expression of promyogenic nuclear factors and cardiomyogenesis of murine embryonic stem cells. Vascul Pharmacol 2014;60:8-16.

14 Grove JE, Bruscia E, Krause DS. Plasticity of bone marrow-derived stem cells. Stem Cells 2004;22:487-500.

15 Cao F, Sun D, Li C, et al. Long-Term myocardial functional improvement after autologous bone marrow mononuclear cells transplantation in patients with ST-segment elevation myocardial infarction: 4 years follow-up. Eur Heart J 2009;30:1986-94.
$16 \mathrm{Hu}$ S, Liu S, Zheng Z, et al. Isolated coronary artery bypass graft combined with bone marrow mononuclear cells delivered through a graft vessel for patients with previous myocardial infarction and chronic heart failure: a single-center, randomized, double-blind, placebo-controlled clinical trial. J Am Coll Cardiol 2011;57:2409-15.

17 Tendera M, Wojakowski W, Ruzyłło W, et al. Intracoronary infusion of bone marrow-derived selected CD34+CXCR4+ cells and nonselected mononuclear cells in patients with acute STEMI and reduced left ventricular ejection fraction: results of randomized, multicentre myocardial regeneration by intracoronary infusion of selected population of stem cells in acute myocardial infarction (regent) trial. Eur Heart J 2009;30:1313-21.

18 Simari RD, Pepine CJ, Traverse JH, et al. Bone marrow mononuclear cell therapy for acute myocardial infarction: a perspective from the cardiovascular cell therapy research network. Circ Res 2014;114:1564-8.

19 Yang Y-J, Qian H-Y, Huang J, et al. Combined therapy with simvastatin and bone marrow-derived mesenchymal stem cells increases benefits in infarcted swine hearts. Arterioscler Thromb Vasc Biol 2009;29:2076-82.

20 Zhang Q, Yang $\mathrm{Y}-\mathrm{J}$, Wang $\mathrm{H}$, et al. Autophagy activation: a novel mechanism of atorvastatin to protect mesenchymal stem cells from hypoxia and serum deprivation via AMP-activated protein kinase/mammalian target of rapamycin pathway. Stem Cells Dev 2012;21:1321-32.

21 Chiang K-H, Cheng W-L, Shih C-M, et al. Statins, HMG-CoA reductase inhibitors, improve neovascularization by increasing the expression density of CXCR4 in endothelial progenitor cells. PLoS One 2015;10:e0136405.

22 Quintavalle C, Fiore D, De Micco F, et al. Impact of a high loading dose of atorvastatin on contrast-induced acute kidney injury. Circulation 2012:126:3008-16.

23 Li N, Yang Y-J, Qian H-Y, et al. Intravenous administration of atorvastatin-pretreated mesenchymal stem cells improves cardiac performance after acute myocardial infarction: role of CXCR4. Am J Transl Res 2015;7:1058-70.

24 Tian X-Q, Yang Y-J, Li Q, et al. Combined therapy with atorvastatin and atorvastatin-pretreated mesenchymal stem cells enhances cardiac performance after acute myocardial infarction by activating SDF-1/CXCR4 axis. Am J Transl Res 2019;11:4214-31.

25 Li Q, Dong Q-T, Yang Y-J, et al. Ampk-Mediated cardioprotection of atorvastatin relates to the reduction of apoptosis and activation of autophagy in infarcted rat hearts. Am J Transl Res 2016;8:4160-71.

26 Xu H, Yang Y-J, Qian H-Y, et al. Rosuvastatin treatment activates JAK-STAT pathway and increases efficacy of allogeneic mesenchymal stem cell transplantation in infarcted hearts. Circ $J$ 2011;75:1476-85.

27 Tossios P, Müller-Ehmsen J, Schmidt M, et al. No evidence of myocardial restoration following transplantation of mononuclear bone marrow cells in coronary bypass grafting surgery patients based upon cardiac SPECT and 18F-PET. BMC Med Imaging 2006;6:7.

28 Hatzistergos KE, Saur D, Seidler B, et al. Stimulatory effects of mesenchymal stem cells on cKit+ cardiac stem cells are mediated by SDF1/CXCR4 and SCF/cKit signaling pathways. Circ Res 2016;119:921-30.

29 Abbott JD, Huang Y, Liu D, et al. Stromal cell-derived factor-1alpha plays a critical role in stem cell recruitment to the heart after myocardial infarction but is not sufficient to induce homing in the absence of injury. Circulation 2004;110:3300-5.

$30 \mathrm{Hu} \mathrm{X}$, Wang J, Chen J, et al. Optimal temporal delivery of bone marrow mesenchymal stem cells in rats with myocardial infarction. Eur J Cardiothorac Surg 2007;31:438-43. 